\title{
Adolescent Idiopathic Scoliosis Treated by Posterior Spinal Segmental Instrumented Fusion : When Is Fusion to L3 Stable?
}

\author{
Seung-Jae Hyun, Lawrence G. Lenke, ${ }^{2}$ Yongjung Kim, ${ }^{3}$ Keith H. Bridwell, ${ }^{4}$ Meghan Cerpa, ${ }^{2}$ Kathy M. Blanke ${ }^{4}$ \\ Department of Neurosurgery, Spine Center, Seoul National University Bundang Hospital, Seoul National University College of Medicine, \\ Seongnam, Korea \\ Department of Orthopaedic Surgery, ${ }^{2}$ Spine Service, Columbia University College of Physicians and Surgeons, New York, NY, USA \\ Department of Orthopaedic Surgery, ${ }^{3}$ Seoul Bumin Hospital, Seoul, Korea \\ Department of Orthopaedic Surgery, ${ }^{4}$ Washington University School of Medicine, St. Louis, MO, USA
}

Objective : The purpose of this study was to identify risk factors for distal adding on (AO) or distal junctional kyphosis (DJK) in adolescent idiopathic scoliosis (AIS) treated by posterior spinal fusion (PSF) to L3 with a minimum 2-year follow-up.

Methods : AIS patients undergoing PSF to L3 by two senior surgeons from 2000-2010 were analyzed. Distal AO and DJK were deemed poor radiographic results and defined as $>3 \mathrm{~cm}$ of deviation from $\mathrm{L} 3$ to the center sacral vertical line (CSVL), or $>10^{\circ}$ angle at L3-4 on the posterior anterior- or lateral X-ray at ultimate follow-up. New stable vertebra (SV) and neutral vertebra (NV) scores were defined for this study. The total stability (TS) score was the sum of the SV and NV scores.

Results : Ten of 76 patients (13.1\%) were included in the poor radiographic outcome group. The other 66 patients were included in the good radiographic outcome group. Lower Risser grade, more SV-3 (CSVL doesn't touch the lowest instrumented vertebra [LIV]) on standing and side bending films, lesser NV and TS score, rigid L3-4 disc, more rotation and deviation of L3 were identified risk factors for AO or DJK. Age, number of fused vertebrae, curve correction, preoperative coronal/sagittal L3-4 disc angle did not differ significantly between the two groups. Multiple logistic regression results indicated that preoperative Risser grade 0, 1 (odds ratio [OR], 1.8), SV-3 at L3 in standing and side benders (OR, 2.1 and 2.8, respectively), TS score $-5,-6$ at $L 3(\mathrm{OR}, 4.4)$, rigid disc at $L 3-4$ (OR, 3.1), LIV rotation $>15^{\circ}(\mathrm{OR}, 2.9)$, and LIV deviation $>2 \mathrm{~cm}$ from $\operatorname{CSVL}(\mathrm{OR}, 2.2)$ were independent predictive factors. Although there was significant improvement of the of Scoliosis Research Society-22 average scores only in the good radiographic outcome group, there was no significant difference in the scores between the groups.

Conclusion : The prevalence of AO or DJK at ultimate follow-up for AIS with LIV at L3 was $13.1 \%$. To prevent AO or DJK following fusion to $\mathrm{L} 3$, we recommend that the CSVL touch L3 in both standing and side bending, TS score is -4 or less, the L3/4 disc is flexible, $L 3$ is neutral $\left(<15^{\circ}\right)$ and $\leq 2 \mathrm{~cm}$ from the midline and the patient is $\geq$ Risser 2 .

Key Words : Adolescent idiopathic scoliosis · Posterior spinal fusion · Lowest instrumented vertebra · Adding-on · Distal junctional kyphosis.

- Received : December 14, 2020 • Accepted : January 5, 2021

- Address for reprints : Seung-Jae Hyun

Department of Neurosurgery, Spine Center, Seoul National University Bundang Hospital, Seoul National University College of Medicine, 82 Gumi-ro 173beon-gil, Bundang-gu, Seongnam 13620, Korea

Tel : +82-31-787-7164, Fax : +82-31-787-4059, E-mail : hyunsj@snu.ac.kr, ORCID : https://orcid.org/0000-0003-2937-5300

This is an Open Access article distributed under the terms of the Creative Commons Attribution Non-Commercial License (http://creativecommons.org/licenses/by-nc/4.0) which permits unrestricted non-commercial use, distribution, and reproduction in any medium, provided the original work is properly cited. 


\section{INTRODUCTION}

Selection of fusion levels is the most important single factor that influences the surgical result following adolescent idiopathic scoliosis (AIS) surgery" ${ }^{5)}$ Inappropriately choosing the extent of fusion may result in under- or overcorrection of the major and compensatory curves. The under- or overcorrection may result in failure to stabilize the index curve and can aggravate the unfused curve and cause trunk imbalance and decompensation. Although surgical correction appears to be relatively straightforward in AIS patients, inadequate selection of fusion levels may cause adding-on (AO) phenomenon and distal junctional kyphosis $(\mathrm{DJK})^{1,4,5,9)}$. Lowest instrumented vertebra (LIV) with rotation more than Nash-Moe Grade II and significant disc angulation below LIV postoperatively and is known as the "AO phenomenon" ${ }^{\sharp, 14,15)}$. Furthermore, for distal fusion level selection in major lumbar and thoracolumbar curves, the selection between L3 or L4 is a debatable issue. $\mathrm{DJK}$ is a junctional angle $>10^{\circ}$ measuring at least $10^{\circ}$ more than the preoperative value. These poor radiographic results including $\mathrm{AO}$ and DJK should be avoided even though we do not have a long-term follow-up study. However, few studies have focused on distal junctional problem, when LIV stopped at L3 for AIS corrective surgery. The purpose of this study was to identify risk factors for poor radiographic results in AIS patients treated by posterior segmental spinal instrumented fu- sion (PSSIF) down to L3 with a minimum two-year follow-up.

\section{MATERIALS AND METHODS}

\section{Demographic and surgical data collection}

After obtaining approval from WASHU Institutional Review Board, extensive review of the patients' medical record was performed to identify demographic, surgical and complication data, including age at surgery, sex, height, weight, curve type by Lenke classification ${ }^{7)}$, number of fused vertebrae, correction rate of the main curve, length of follow-up. For clinical outcome evaluation, Scoliosis Research Society (SRS)-22 questionnaires score was investigated.

\section{Patient population}

Inclusion criteria were as follows : 1) any AIS patients treated with posterior pedicle screw constructs, 2) the LIV at L3, and 3) with a minimum 2-year follow-up. Patients with neuromuscular disease or congenital spinal deformity, and those underwent revision surgery were excluded. Seventy-six consecutive AIS patients between 2000 and 2010 who met the inclusion criteria were identified from a single institution database. The 76 patients consisted of 70 girls and six boys. Mean age at surgery was 14.7 years old (ranged from 10.0 to 19.6). Average follow-up period was 3.2 years (range, 2.0-10.2). All

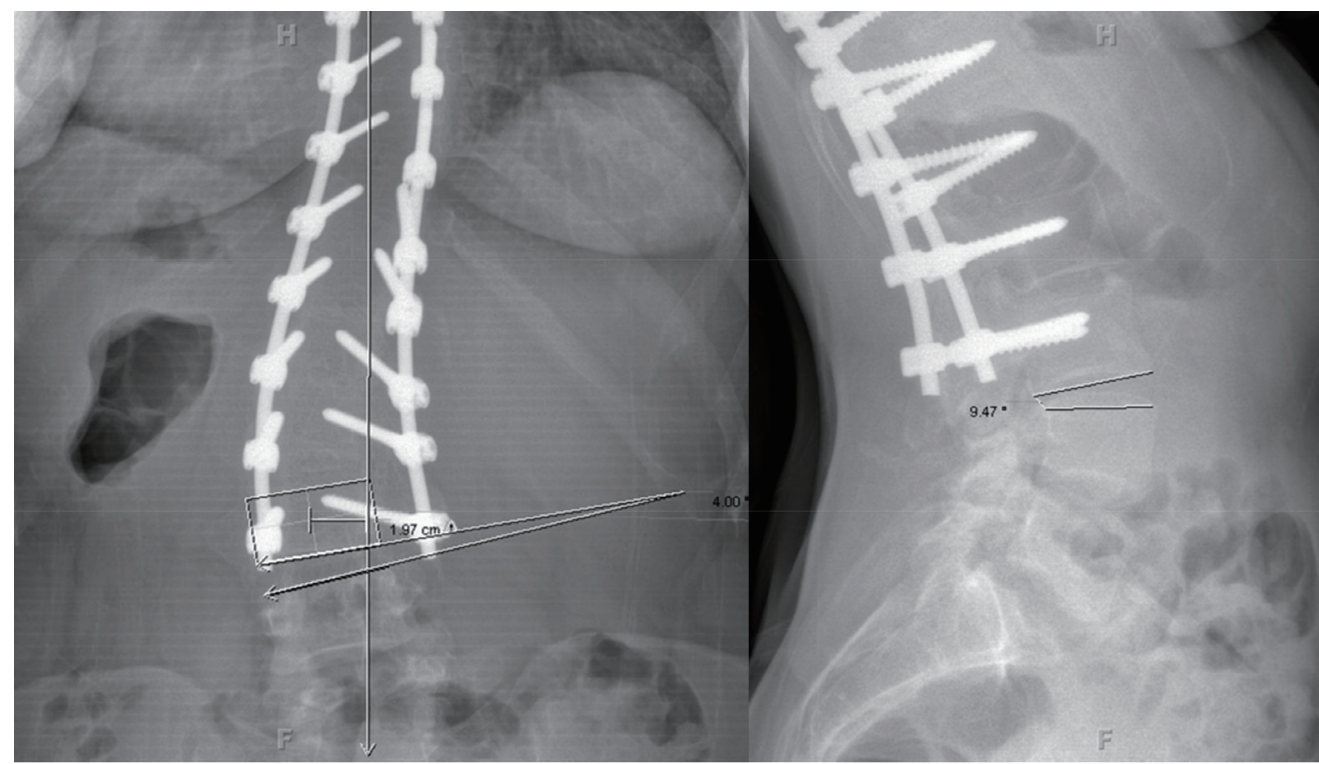

Fig. 1. An example of radiographic measurement for deviation of the center of the $L 3$ from the center sacral vertical line, distal junctional discal angulation at L3-4 in the coronal or sagittal plane. 
enrolled patients were surgically treated by two senior attending surgeons (L.G.L. and K.H.B.).

\section{Radiographic measurements}

Measurements were made on upright posterior anterior, side bending and lateral radiographs of the entire spine. Distal $\mathrm{AO}$ was defined as a progressive increase in the number of vertebrae included distally within the primary curve combined with either an increase of more than $3 \mathrm{~cm}$ in deviation of the center of the LIV from the center sacral vertical line (CSVL), or an increase of more than $10^{\circ}$ in the coronal angulation of the first disc below the instrumentation at ultimate

Table 1. Definition of gravity, rotational and total stability score

\begin{tabular}{|c|c|}
\hline & Definition \\
\hline $\begin{array}{l}\text { Gravity stability } \\
\text { score }\end{array}$ & $\begin{array}{l}\text { SV : LIV at SV } \\
\text { SV-1: CSVL passes between medial pedicle borders } \\
\text { of the LIV } \\
\text { SV-2: CSVL touches the LIV } \\
\text { SV-3: CSVL does not touch the LIV }\end{array}$ \\
\hline $\begin{array}{l}\text { Rotational } \\
\text { stability score }\end{array}$ & $\begin{array}{l}\text { NV : LIV at NV } \\
\text { NV-1 : LIV is one vertebra proximal to NV } \\
\text { NV-2 : LIV is two vertebra proximal to NV } \\
\text { NV-3 : LIV is three vertebra proximal to NV }\end{array}$ \\
\hline $\begin{array}{l}\text { Total stability } \\
\text { score }\end{array}$ & TS-0 to -6 : Summation of SV and NV score \\
\hline
\end{tabular}

SV : stable vertebra, LIV : lowest instrumented vertebra, CSVL : center sacral vertical line, NV : neutral vertebra, TS : total stability follow-up. DJK was defined if sagittal disc angle below the LIV is more than $10^{\circ}$. In this study, poor radiographic outcomes were defined as the distance from CSVL to the center of $\mathrm{L} 3 \geq 3 \mathrm{~cm}$, or a discal angle at L3-4 $>10^{\circ}$ in the coronal or sagittal plane at ultimate follow-up.

Investigated radiographic parameters included : Risser grade, correction rate, preoperative coronal rotation angle using Perdriolle method $^{12)}$ and deviation distance of L3, coronal and sagittal disc angle at L3-4 (Fig. 1), gravity stability score in standing and side bender (new stable vertebra [SV] was defined for this study : SV-1, CSVL is passing between medial borders of pedicles of the LIV; SV-2, CSVL touching body of LIV; SV-3, CSVL does not touch LIV body), rotational stability score (neutral vertebra $[\mathrm{NV}]$ : vertebra without rotation; NV-1, one vertebra proximal to NV; NV-2, two vertebra proximal to NV; NV-3, three vertebra proximal to NV), and total stability score (summation of gravity and rotational stability score) (Table 1 and Fig. 2).

\section{Assessment of disc flexibility at L3-4}

L3-4 disc angle was measured between straight lines along the inferior endplate of the upper and the superior endplate of the lower vertebra in a segment. This was done on the upright and side bending radiographs. The following equation ${ }^{2)}$ was used for the disc flexibility at L3-4 :

Disc flexibility index $(\%)=$ (upright disc angle - bending disc angle $) \times 100 /$ upright disc angle

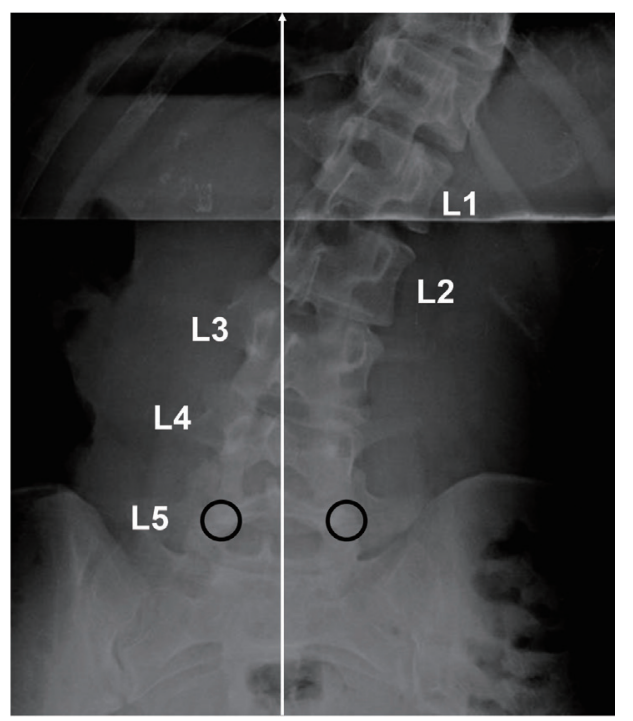

\begin{tabular}{|c|c|c|c|}
\hline & Gravity stability & Rotational stability & Total stability \\
\hline L1 & SV-3 & NV-3 & TS-6 \\
\hline L2 & SV-2 & NV-3 & TS-5 \\
\hline L3 & SV-1 & NV-2 & TS-3 \\
\hline L4 & SV & NV-1 & TS-1 \\
\hline L5 & SV & NV & TS-0 \\
\hline
\end{tabular}

Fig. 2. An example of radiographic evaluation for gravity, rotational and total stability scoring system. SV : stable vertebra, NV : neutral vertebra, TS : total stability. 
When the disc flexibility index was more than $25 \%$, the L3-4 disc was defined as flexible. Similarly, rigid disc at L3-4 was defined if the disc flexibility index was less than $25 \%$.

\section{Statistical analysis}

Distributions of variables were given as a mean \pm standard deviation. For most variables for which data was collected preoperatively and postoperatively, paired t-tests were used to determine whether there was a significant change between time- points. Student t-test was used to assess the difference of continuous measures between the groups. Fisher's exact test was used for dichotomous data analysis depending on the number of subjects involved. Multiple logistic regression test was used to identify the risk factors and odds ratio for poor radiographic outcomes including $\mathrm{AO}$ or DJK. A $p$-value of $<0.05$ was considered statistically significant.

Table 2. Clinical and radiographic factors between patients with good or poor radiographic outcomes

\begin{tabular}{|c|c|c|c|}
\hline & GX group $(n=66)$ & PX group $(n=10)$ & $p$-value \\
\hline \multicolumn{4}{|l|}{ Demographic data } \\
\hline Age at surgery (years) & $14.9 \pm 2.0$ & $13.6 \pm 2.0$ & 0.642 \\
\hline F/U duration (years) & $3.1 \pm 2.0$ & $3.7 \pm 2.6$ & 0.380 \\
\hline Risser grade & $3.8 \pm 1.4$ & $2.2 \pm 2.0$ & 0.003 \\
\hline \multicolumn{4}{|l|}{ Preoperative radiographic factor } \\
\hline Gravity stability score (SV) & $-2.3 \pm 0.6$ & -3.0 & 0.004 \\
\hline Rotational stability score (NV) & $-1.2 \pm 1.0$ & $-1.8 \pm 0.6$ & 0.024 \\
\hline TS score & $-3.6 \pm 1.5$ & $-4.8 \pm 0.6$ & $<0.001$ \\
\hline Rigid disc at $L 3 / 4$ & 1 & 3 & $<0.001$ \\
\hline LTV at L3 on upright standing & $34(56.7)$ & $0(0.0)$ & 0.001 \\
\hline LTV at L3 on side benders & $66(100.0)$ & $7(70.0)$ & 0.081 \\
\hline Rotation angle of $\mathrm{L} 3$ & $7.4 \pm 5.2$ & $16.5 \pm 8.8$ & 0.005 \\
\hline Distance from CSVL to L3 & $-1.8 \pm 0.9$ & $-3.1 \pm 0.9$ & $<0.001$ \\
\hline Coronal disc angle at $\mathrm{L} 3 / 4$ & $5.4 \pm 2.5$ & $4.5 \pm 1.4$ & 0.234 \\
\hline Sagittal disc angle at $L 3 / 4$ & $-8.3 \pm 2.0$ & $-8.1 \pm 3.7$ & 0.993 \\
\hline \multicolumn{4}{|l|}{ Postoperative radiographic factor } \\
\hline Number of fused vertebra & $11.5 \pm 2.6$ & $10.9 \pm 2.3$ & 0.437 \\
\hline Correction rate of major curve (\%) & $78.5 \pm 7.3$ & $79.1 \pm 6.9$ & 0.521 \\
\hline
\end{tabular}

Values are presented as mean \pm standard deviation or number (\%). GX: good poor radiographic outcome, PX: poor radiographic outcome, F/U : followup, SV : stable vertebra, NV : neutral vertebra, TS : total stability, LTV : last touching vertebra, CSVL : center sacral vertical line

Table 3. Identified risk factors associated with poor radiographic outcomes by multiple regression analysis

\begin{tabular}{lccc}
\hline & Odds ratio & Confidence interval & $p$-value \\
\hline Risser grade 0,1 & 1.8 & $1.4-2.3$ & 0.014 \\
SV-3 at L3 in upright & 2.1 & $1.7-2.7$ & 0.003 \\
\hline SV-3 at L3 in side benders & 2.8 & $1.9-3.6$ & $<0.001$ \\
Total stability score $-5,-6$ & 4.4 & $2.8-6.3$ & $<0.001$ \\
\hline Rigid disc at L3/4 & 3.1 & $2.3-3.9$ & $<0.001$ \\
L3 rotation $>15^{\circ}$ & 2.9 & $2.2-3.7$ & 0.001 \\
\hline L3 deviation $>2$ cm from CSVL & 2.2 & $1.7-2.7$ & 0.006 \\
\hline
\end{tabular}

SV-3 means CSVL does not touch the index vertebra. CSVL indicates center sacral vertical line. SV : stable vertebra, CSVL : center sacral vertical line 


\section{RESULTS}

Ten of 76 patients $(13.1 \%)$ were included in the poor radiographic outcome group. The remaining 66 patients were included in the good radiographic outcome group. Patient demographic data of both groups are summarized in Table 2. Age at surgery and follow-up duration did not show differences between the groups. However, Risser grade was significantly lower in the poor radiographic outcome groups $(p=0.003)$

\section{Radiographic factors}

Poor radiographic outcomes group showed lesser gravity, rotational and total stability score $(p=0.004, p=0.024$, and $p<0.001$, respectively), more rigid disc at L3 $-4(p<0.001)$, more rotation $(p=0.005)$, and more deviation from CSVL $(p<0.001)$ (Table 2). Last touching vertebra (LTV) was always at L4 in the poor radiographic outcome group. Thirty-four patients (56.7\%) had LTV at L3 on upright standing in the good radiographic outcome group. Patients having LTV at L3 on side benders was more frequent in the good radiographic outcome group (100\% vs. $70 \%)$. Number of fused vertebrae, correction rate of the major curve, preoperative coronal and sagittal junctional disc angle at L3-4 did not show significant differences between the groups.

Multiple logistic regression results indicated that preoperative Risser grade 0, 1 (odds ratio [OR], 1.8; 95\% confidence interval $[\mathrm{CI}], 1.4-2.3 ; p=0.014), \mathrm{SV}-3$ at L3 in standing and side benders (OR, 2.1; 95\% CI, 1.7-2.7; $p=0.003$ and OR, 2.8; 95\% CI, 1.9-3.6; $p<0.001$, respectively), total stability score $-5,-6$ at L3 (OR, 4.4; 95\% CI, 2.8-6.3; $p<0.001)$, rigid disc at L3-4 (OR, 3.1; 95\% CI, 2.3-3.9; $p<0.001$ ), LIV rotation $\geq 15^{\circ}$ (OR, 2.9; 95\% CI, 2.2-3.7; $p=0.001$ ), and LIV deviation $>2 \mathrm{~cm}$ from CSVL (OR, 2.2; 95\% CI, 1.7-2.7; $p=0.006$ ) were independent predictive factors associated with poor radiographic outcomes
(Table 3).

\section{Clinical outcomes}

Any patient did not undergo revision surgery in the both groups. Although there was a significant improvement ( $p=0.023$ ) of the average scores of SRS-22 questionnaires only in the good radiographic outcome group, there was no significant difference of the scores of SRS-22 questionnaires between the groups (Table 4).

\section{DISCUSSION}

Optimal LIV to avoid AO or DJK is extremely idiosyncratic. Various concepts and rules were introduced by previous researchers such as Harrington stable zone, SV and NV theory, disc reversal and $\mathrm{LTV}^{1,3-6,9-11,13)}$. However, poor inter-rater reliability for LIV selection was reported even among 17 SRS surgeons $^{8)}$. In their study, 50\% agreement was observed and Kappa value was 0.38 (poor reliability). Therefore, this study was aimed to identify risk factors for the presence of distal AO or DJK in AIS treated by PSSIF caudal to L3 with a minimum two-year follow-up. In this series, the prevalence of AO or DJK at ultimate follow-up with LIV at L3 was 13.1\%. The prevalence of $\mathrm{AO}$ or DJK is similar to a study focusing the prevalence $(13.6 \%)$ of $\mathrm{AO}$ or DJK following PSSIF for AIS with LIV at L2 or above ${ }^{4)}$. In their study, open tri-radiate cartilage, not touching of the LIV by the CSVL, and more rotation of the LIV were identified as risk factors for $\mathrm{AO}$ or DJK.

In the present study, lower Risser grade, more SV-3 on standing and side bending films, lesser rotational and total stability score, rigid L3-4 disc, more rotation and deviation of L3 were identified risk factors for AO or DJK (Fig. 3). Furthermore, multiple logistic regression results indicated that preop-

Table 4. Summary of clinical outcomes

\begin{tabular}{lccc}
\hline & GX group $(\mathbf{n}=66)$ & PX group $(\mathbf{n}=10)$ & \\
\hline SRS-22 average scores & & & \\
Preoperative & $4.06 \pm 1.35$ & $4.30 \pm 0.43$ & 0.317 \\
Ultimate follow-up & $4.62 \pm 1.17$ & $4.37 \pm 0.49$ & 0.541 \\
$p$-value & 0.023 & 0.752 & \\
\hline
\end{tabular}

Values are presented as mean \pm standard deviation. GX : good radiographic outcome, PX : poor radiographic outcome, SRS-22 : scoliosis research society-22 questionnaire 
erative Risser grade 0, 1 (OR, 1.8), SV-3 at L3 in standing and side benders (OR, 2.1 and 2.8, respectively), total stability score 5,6 at $\mathrm{L} 3(\mathrm{OR}, 4.4)$, rigid disc at $\mathrm{L} 3-4(\mathrm{OR}, 3.1)$, LIV rotation $>15^{\circ}$ (OR, 2.9), and LIV deviation $>2 \mathrm{~cm}$ from CSVL (OR, 2.2) were significant predictive factors for poor radiographic outcomes. For these analyses, we utilized a new gravity, rotational and total stability scoring system. In our new scoring system, the difference between SV-2 and SV-3 is whether CSVL does touch LIV or not. It means that SV-2 and SV-1 is LTV and substantial LTV, respectively. Total stability score is the sum of gravity and rotational stability score. By the multiple logistic regression analysis, total stability score -5 or less at L3 (OR, 4.4) is the most significant factor associated with poor radiographic outcomes after stopping at L3. To the best of our knowledge, there are no published reports using gravity, rotational and total stability scoring system to determine optimal LIV level.

In major thoracolumbar of lumbar structural curves, it had been considered that fusion should be extended down to L4 in the era of Harrington instrumentation ${ }^{1,13,15,16)}$. However, stop- ping at L3 instead of L4 has been proposed in the era of segmental pedicle screw based instrumentation. Lenke and colleagues ${ }^{8)}$ proposed the criteria for stopping of distal fusion at L3, as follows : 1) less than Nash-Moe grade I rotation of L3; 2) tilt of $\mathrm{L} 3<30^{\circ}$ and tilt of $\mathrm{L} 4<20^{\circ}$; 3) L4 vertebra body was bisected by the CSVL; 4) apical disc should be located at T12L1 or above; 5) the direction of opening at the L3-L4 level should be parallel to or opposite the L4-L5 disc level; and 6) the location of L3 should be centered by bending. Recently, selecting the LTV by CSVL as an optimal LIV can decrease the incidence of distal $\mathrm{AO}^{10,13,15)}$. The previously reported factors or criteria are valuable to determine distal fusion levels in AIS. However, absolute guidelines for the selection of LIV have not been defined. In the current study, we found several key risk factors for $\mathrm{AO}$ or DJK. Moreover, we introduce the odds ratio of each risk factor by multiple logistic regression analysis. We can share and discuss the information of predicting factors for poor radiographic outcomes with AIS kids and their guardians.
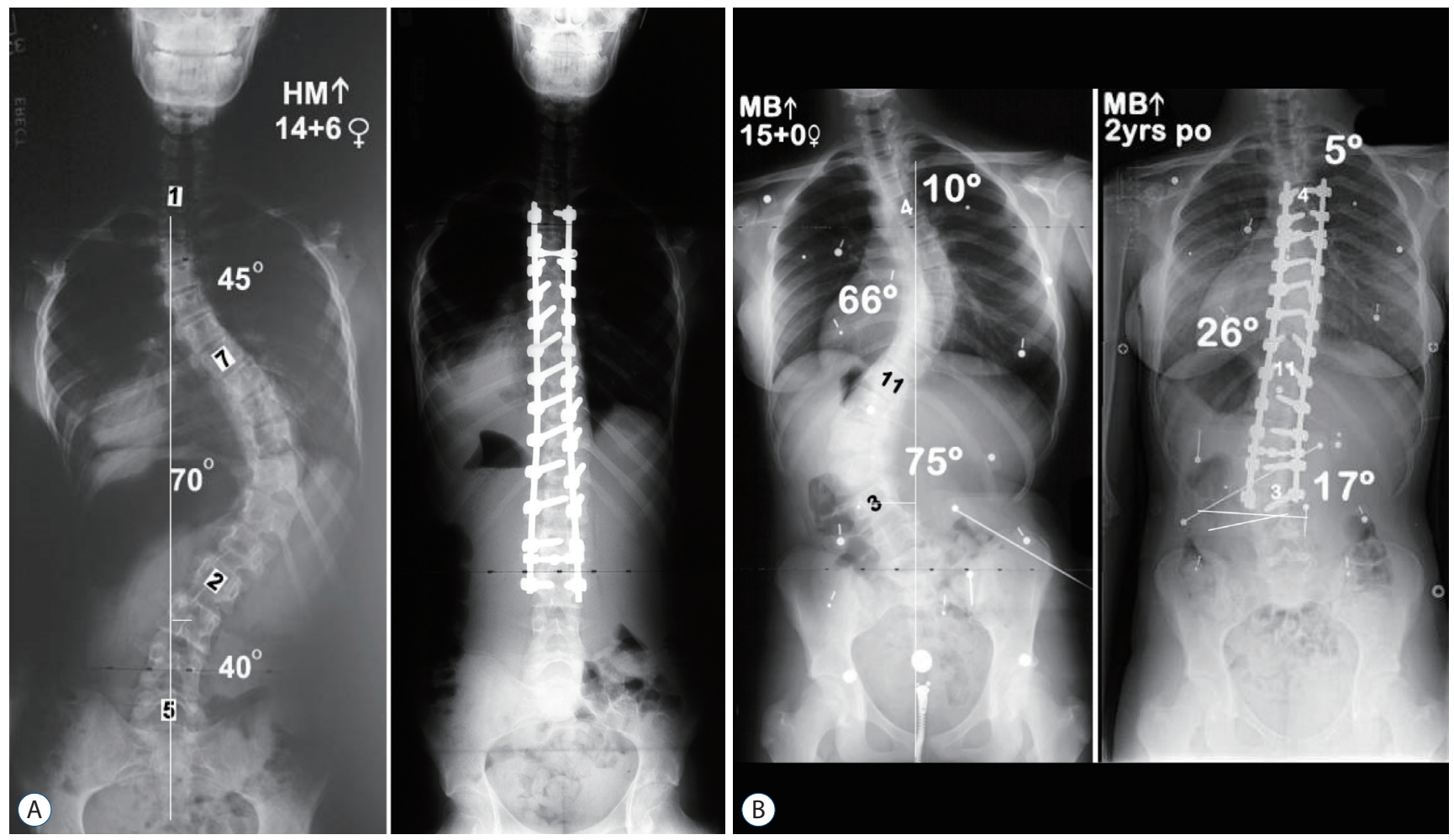

Fig. 3. Representative cases of good (A) and poor (B) radiographic outcomes. A : A 14-year-old girl having neutral $\left(<15^{\circ}\right) \mathrm{L} 3$ touched by the center sacral vertical line (CSVL) and located within $2 \mathrm{~cm}$ from the midline shows good radiographic outcome. B : A 15-year-old girl having rotated (>15 $)$ L3 not touched by the CSVL and deviated more than $2 \mathrm{~cm}$ from the midline demonstrates poor radiographic outcome with $13^{\circ}$ distal junctional discal angulation at L3-4 in the coronal plane. po : postoperative. 


\section{CONCLUSION}

The prevalence of $\mathrm{AO}$ or DJK at ultimate follow-up with LIV at L3 was $13.1 \%$. To prevent $\mathrm{AO}$ or DJK following posterior spinal fusion caudal to L3, the CSVL should touch L3 on standing and side bending films, the distal junctional disc should be flexible, L3 should be neutral $\left(<15^{\circ}\right)$ and $\geq 2 \mathrm{~cm}$ from the midline, and the patient should be Risser grade 2 or greater.

\section{CONFLICTS OF INTEREST}

No potential conflict of interest relevant to this article was reported.

\section{INFORMED CONSENT}

Informed consent was obtained from all individual participants included in this study.

\section{AUTHOR CONTRIBUTIONS}

\author{
Conceptualization : SJH, YK \\ Data curation : SJH, YK, LGL, KHB, KMB \\ Formal analysis: $\mathrm{SJH}, \mathrm{YK}$ \\ Methodology : SJH, YK \\ Project administration : SJH, YK, LGL, KHB, KMB \\ Visualization : $\mathrm{SJH}$ \\ Writing - original draft : SJH \\ Writing - review \& editing : SJH, YK, MC, KMB
}

\section{ORCID}

Seung-Jae Hyun https://orcid.org/0000-0003-2937-5300

Lawrence G. Lenke https://orcid.org/0000-0002-5595-4958

Yongjung Kim https://orcid.org/0000-0003-1647-3286

Keith H. Bridwell https://orcid.org/0000-0001-9228-8782

Meghan Cerpa https://orcid.org/0000-0002-5931-7067

Kathy M. Blanke https://orcid.org/0000-0002-7748-0693

\section{- Acknowledgements}

Research was performed at Washington University School of Medicine.

\section{References}

1. Harrington PR : Treatment of scoliosis. Correction and internal fixation by spine instrumentation. J Bone Joint Surg Am 44-A : 591-610, 1962

2. Hasler $C C$, Hefti F, Büchler $P$ : Coronal plane segmental flexibility in thoracic adolescent idiopathic scoliosis assessed by fulcrum-bending radiographs. Eur Spine J 19 : 732-738, 2010

3. Kim CW, Hyun SJ, Kim KJ : Surgical impact on global sagittal alignment and health-related quality of life following cervical kyphosis correction surgery: systematic review. Neurospine 17 : 497-504, 2020

4. Kim YJ, Fischer CR, Lenke LG, Bridwell KH, Boachie-Adjei O, Clement $J$, et al. : Optimal lowest instrumented vertebra to avoid adding-on or distal junctional kyphosis for thoracic adolescent idiopathic scoliosis. Scoliosis Research Society 46th Annual Meeting; 2011 Sep 1417; Louisville, KY. Podium presentation 88

5. King HA, Moe JH, Bradford DS, Winter RB : The selection of fusion levels in thoracic idiopathic scoliosis. J Bone Joint Surg Am 65 : 1302-1313, 1983

6. Lee $\mathrm{SH}$, Hyun SJ, Jain A : Cervical sagittal alignment: literature review and future directions. Neurospine $17:$ 478-496, 2020

7. Lenke LG, Betz RR, Harms J, Bridwell KH, Clements DH, Lowe TG, et al. : Adolescent idiopathic scoliosis: a new classification to determine extent of spinal arthrodesis. J Bone Joint Surg Am 83 : 1169-1181, 2001

8. Lenke LG, Braun JT, Bridwell KH, Dimar JR, Dobbs M, Glassman S, et al. : Reliability analysis of end, neutral, stable, and lowest instrumented vertebrae selection in adolescent idiopathic scoliosis: Surgeon versus digital software determination. 12th International Meeting on Advanced Spine Techniques; 2005 Jul 7-9; Banff, Canada. Podium presentation 46

9. Lenke LG, Bridwell KH, Baldus C, Blanke K, Schoenecker PL : Ability of Cotrel-Dubousset instrumentation to preserve distal lumbar motion segments in adolescent idiopathic scoliosis. J Spinal Disord 6 : 339-350, 1993

10. Lenke LG, Newton PO, Lehman RA, Kelly MP, Clements DH, Errico TJ, et al. : Radiographic results of selecting the touched vertebra as the lowest instrumented vertebra in Lenke $1 \mathrm{~A}$ AIS curves at a minimum fiveyear follow up. Scoliosis Research Society 49th Annual Meeting; 2014 Sep 11-13; Anchorage, AK. Podium presentation 5

11. Park BJ, Hyun SJ, Wui SH, Jung JM, Kim KJ, Jahng TA : Surgical outcomes and complications following all posterior approach for spinal deformity associated with neurofibromatosis type-1. J Korean Neurosurg Soc 63 : 738-746, 2020

12. Perdriolle R, Vidal J : Morphology of scoliosis: three-dimensional evolu- 
When Is Fusion to L3 Stable? I Hyun SJ, et al.

tion. Orthopedics $10: 909-915,1987$

13. Qin X, Sun W, Xu L, Liu Z, Qiu Y, Zhu Z : Selecting the last "substantially" touching vertebra as lowest instrumented vertebra in Lenke type 1 A curve: radiographic outcomes with a minimum of 2-year follow-up. Spine (Phila Pa 1976) 41 : E742-E750, 2016

14. Suk SI, Lee SM, Chung ER, Kim JH, Kim WJ, Sohn HM : Determination of distal fusion level with segmental pedicle screw fixation in single thoracic idiopathic scoliosis. Spine (Phila Pa 1976) 28 : 484-491, 2003
15. Wang Y, Hansen ES, Høy K, Wu C, Bünger CE : Distal adding-on phenomenon in Lenke $1 A$ scoliosis: risk factor identification and treatment strategy comparison. Spine (Phila Pa 1976) 36 : 1113-1122, 2011

16. Wui SH, Hyun SJ, Kang B, Kim KJ, Jahng TA, Kim HJ : Bicortical screw purchase at upper instrumented vertebra (UIV) can cause UIV fracture after adult spinal deformity surgery: a finite element analysis study. Neurospine 17 : 377-383, 2020 